\title{
Equilibrium shapes of nonaxisymmetric liquid bridges of arbitrary volume in gravitational fields and their potential energy
}

\author{
Ana Laverón-Simavilla and José M. Perales \\ E.T.S.I. Aeronáuticos, Universidad Politécnica de Madrid, 28040 Madrid, Spain
}

\begin{abstract}
Bifurcation diagrams of nonaxisymmetric liquid bridges subject to a lateral gravitational force and to both fateral and axial gravitational forces are found by solving the Young-Laplace equation for the interface by a finite difference method. The potential energy of the equilibrium shapes is also calculated. The results obtained show that the slenderness of the bridge determines whether the breaking of the liquid bridge subject to a lateral gravitational force leads to equal or unequal drops. The stability limits calculated are compared with the ones obtained using asymptotic techniques around the cylinder, the agreement being extremely good for a wide range of the parameters.
\end{abstract}

\section{INTRODUCTION}

The floating zone method is a technique used in fabricating ultrapure semiconductor crystals. The mechanical aspects of the configuration can be modeled by the so-called liquid bridge, which consists of a mass of liquid held by surface tension forces between two circular disks. The first studies on static shapes of liquid bridges held between two coaxial cylindrical disks dates back to the past century, ${ }^{1}$ but recent availability of fluid mechanics experiments in space has increased the interest in the problem.

Most of the results obtained up to now refer to axisymmetric liquid bridges, even though in some occasions it has been considered that the perturbations affecting the stability could be nonaxisymmetric. The consideration of the axisymmetry of the initial equilibrium shape has simplified in a great deal the original problem and therefore a method to determine the stability limit has been developed ${ }^{2}$ (recent results can be found in Slobozhanin and Perales ${ }^{3}$ ). There exists some asymptotic studies about nonaxisymmetric liquid bridges, namely the influence on the stability of an almost cyindrical liquid bridge subject to nonaxisymmetric perturbations, such as a lateral acceleration and noncoaxial supporting disks, has been analyzed using standard perturbation techniques. The small deformation of the liquid bridge interface associated to each of the mentioned perturbations has also been obtained. ${ }^{4-6}$ Some asymptotic results have also been obtained concerning nonaxisymmetric instability, which appears when a cylindrical liquid bridge is rotated as a solid body. ${ }^{7.8}$

A finite element algorithm employing Newton iteration and a block-Lanczos method was applied to gyrostatic liquid bridges of fixed volume held between two corotating, parallel, concentric supporting circular disks, to obtain the family of solutions generated by varying the rotation and to determine their stability. The results for the special case of captive cylindrical drops were compared with published results and with new results obtained by conventional stability bifurcation analysis. ${ }^{9}$ The bifurcation diagrams and the stability limits of inclined sessile and pendant drops have also been obtained developing a finite element model. ${ }^{10,11}$

In a very recent work nonaxisymmetric equilibrium shapes of two typical problems have been obtained; the first is a short liquid bridge inclined relative to the direction of gravity in a terrestrial environment, and the second is a long liquid bridge, which is subject to a lateral gravitational force in microgravity environment. The solutions were obtained for a set of parameters using a finite element program. ${ }^{12}$

The aim of this paper is to present an algorithm capable of calculating the sequence of nonaxisymmetric equilibrium shapes obtained by varying a parameter of the configuration and the potential energy of each one of the calculated shapes.

The developed algorithm is based on a continuation method that is capable of overpassing bifurcation points, and is specially efficient for overpassing turning points. ${ }^{13}$ To solve the Young-Laplace equation, a method of finite differences has been developed constructing a difference scheme that approximates the continuous problem. To do so, the domain of the continuous variation of the arguments has been replaced by a net domain, which is a set of points called nodal points.

\section{FORMULATION}

The fluid configuration considered consists of a mass of liquid of volume $\mathscr{F}^{\prime}$ held by surface tension forces between two paralleI, coaxial, circular disks of radii $R$, placed a distance $L$ apart. The liquid bridge is subjected to a gravitational field, as sketched in Fig. 1. Even though, for simplicity, the noncircularity of the disks has not been taken into account, it is easy to extend the problem in order to consider the case in which the disks are not circular.

The equation governing the steady shape of the liquid bridge is obtained by expressing the equilibrium between the surface tension forces and the local pressure forces at the free surface of the liquid. This equation can be written using dimensionless parameters and variables, as follows:

$$
M(F, X, Y)+P-B_{a} z+B_{l}(X+F \cos \theta)=0,
$$

where all the lengths have been made dimensionless with the radius of the disks, $R, \mathrm{x}(z, \theta)=\{X(z)+F(z, \theta) \cos \theta, Y(z)$ $+F(z, \theta) \sin \theta, z\}$ is the dimensionless parametric equation of the free surface, where $X(z)$ and $Y(z)$ are introduced in order to avoid multivaluate solutions, $P$ is a dimensionless 
constant defined as $P=R\left(P_{c}-P_{o}\right) / \sigma$ (where $\sigma$ is the surface tension coefficient, $P_{c}$ is the inner reference pressure, and $P_{a}$ is the outer pressure, which is assumed to be constant), $B_{a}$ is the axial Bond number defined as $B_{a}=\rho\left|\mathbf{g}_{a}\right| R^{2} / \sigma$ (where $\mathbf{g}_{a}$ is the axial component of the gravity, and $\rho$ is the density difference between the liquid and the outer fluid), $B_{1}$ is the lateral Bond number defined as $B_{l}=\rho\left|\mathbf{g}_{l}\right| R^{2} / \sigma$ (where $\mathrm{g}_{l}$ is the lateral component of the gravity), and, finally, twice the local mean curvature of the free surface, $M(F, X, Y)$, is given by

$$
\begin{aligned}
M(F, X, Y)= & \left\{\left[1+\left(X_{z}+F_{z} \cos \theta\right)^{2}+\left(Y_{z}+F_{z} \sin \theta\right)^{2}\right] \cdot\left(F^{2}+2 F_{\theta}^{2}-F F_{\theta \theta}\right)+\left(F^{2}+F_{\theta}^{2}\right) \cdot\left[-X_{z z}\left(F_{\theta} \sin \theta+F \cos \theta\right)\right.\right. \\
& \left.+Y_{z z}\left(F_{\theta} \cos \theta-F \sin \theta\right)-F F_{z z}\right]-2\left(F_{z} F_{\theta}-F F_{z \theta}\right) \cdot\left[F_{z} F_{\theta}+X_{z}\left(F_{\theta} \cos \theta-F \sin \theta\right)\right. \\
& \left.\left.+Y_{z}\left(F_{\theta} \sin \theta+F \cos \theta\right)\right]\right\} \cdot\left\{\left[1+\left(X_{z}+F_{z} \cos \theta\right)^{2}+\left(Y_{z}+F_{z} \sin \theta\right)^{2}\right]\left(F^{2}+F_{\theta}^{2}\right)-\left[X_{z}\left(F_{\theta} \cos \theta-F \sin \theta\right)\right.\right. \\
& \left.\left.+Y_{z}\left(F_{\theta} \sin \theta+F \cos \theta\right)+F_{z} F_{\theta}\right]^{2}\right\}^{-3 / 2}
\end{aligned}
$$

Equation (1) has to be integrated with the boundary conditions

$$
\begin{aligned}
& F( \pm \Lambda, \theta)=1, \\
& X( \pm \Lambda)=0, \\
& Y( \pm \Lambda)=0, \\
& F(z, \theta)=F(z, \theta+2 \pi), \\
& F(z, 0)=F(z, \pi), \\
& F(z, \pi / 2)=F(z, 3 \pi / 2), \\
& \int_{-\Lambda}^{\Lambda} d z \int_{0}^{2 \pi} F^{2}(z, \theta) d \theta=4 \pi \Lambda V,
\end{aligned}
$$

where $\Lambda$ is the slenderness of the liquid bridge defined as $\Lambda=L /(2 R)$, and $V$ is the dimensionless volume defined as

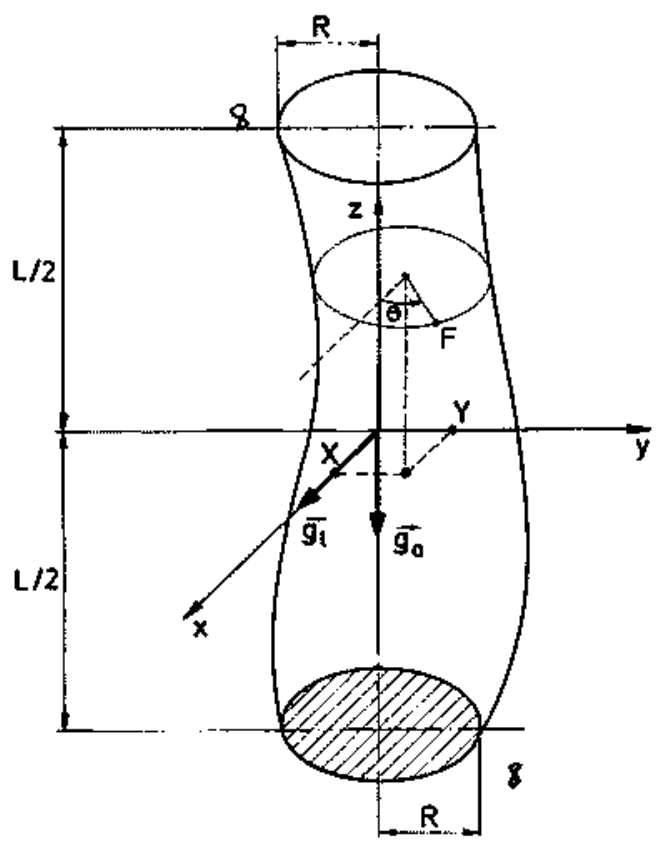

FIG. 1. Geometry and coordinate system for the liquid bridge configuration considered.
$V=\mathscr{g} /\left(2 \pi \Lambda R^{3}\right)$. Equation (3) indicates that the free surface is anchored to the sharp edges of the disks, Eqs. (4) and (5) indicate the position of the disks, Eq. (6) comes from the azimuthal periodicity, Eqs. (7) and (8) uniquely determine the auxiliary functions $X(z)$ and $Y(z)$, respectively, and Eq. (9) is the condition of conservation of the volume of the liquid bridge. Taking into account the existing symmetry in the configuration with respect to the plane $y=0$, it is possible to choose $Y(z)=0$ so that Eqs. (5) and (8) are no longer necessary.

The system of equations (1)-(9) is nonlinear and a finite difference method is used to solve it. Considering the problem in parametric formulation, the resulting discrete equations can be written as

$$
G(u, \lambda)=0, \quad G: \mathrm{R}^{N} \times \mathbb{R} \rightarrow \mathbf{R}^{N},
$$

where $u=\{F, X, P\}$ and $\lambda$ is one of the parameters of the configuration. Assuming that a solution is known, Newton's method can be accomplished to compute a path of solutions progressing with the natural parameter $\lambda$, until a fold point is encountered. At fold points Newton's method fails during natural parameter continuation because the Jacobian matrix, $G_{u}(u, \lambda)$, is singular at those points, therefore an auxiliary parameter, $s$, is introduced, so that the unknowns are supplemented ${ }^{13}$ with the natural parameter $\lambda$. However, a new equation is needed to be adjoined to Eq. (10),

$$
\begin{aligned}
N_{1}(u, \lambda, s)= & \dot{u}^{T}\left(s_{o}\right)\left[u(s)-u\left(s_{o}\right)\right]+\dot{\lambda}\left(s_{o}\right)[\lambda(s) \\
& \left.-\lambda\left(s_{o}\right)\right]-\Delta s=0,
\end{aligned}
$$

where

$$
\dot{u}^{T}\left(s_{o}\right) \dot{u}\left(s_{o}\right)=\left\|\dot{u}\left(s_{o}\right)\right\|^{2},
$$

being $\left[u\left(s_{o}\right), \lambda\left(s_{o}\right)\right]$ a previously known solution and $\left[\dot{u}\left(s_{o}\right), \dot{\lambda}\left(s_{o}\right)\right]$ the unit tangent vector to the solution path in the mentioned known solution. The system of Eqs. (10) and (11) can be rewritten as

$$
\mathscr{F}(w, s)=0, \quad \mathscr{F}: \mathbf{R}^{N+1} \times \mathbf{R} \rightarrow \mathbb{R}^{N+1},
$$

where $w=(u, \lambda)$ and

$$
\oiint(w, s)=\left\{\begin{array}{c}
G(u, \lambda) \\
N_{1}(u, \lambda, s)
\end{array}\right\} .
$$


The continuation algorithm can be written as

$$
\begin{aligned}
& s=s_{o}+\delta s_{o}, \\
& w^{0}=w_{o}+\delta w_{o}, \\
& F_{w}\left(w_{o}, s_{o}\right) \delta w_{o}=-\mathscr{F}_{s}\left(w_{o}, s_{o}\right) \delta s_{o},
\end{aligned}
$$

for the initial guess, and

$$
\begin{aligned}
& w^{\nu+1}=w^{\nu}+\delta w^{\nu}, \quad \nu=0,1,2, \ldots, \\
& \mathscr{F}_{w}\left(w^{\nu}, s\right) \delta w^{\nu}=-\mathscr{F}\left(w^{\nu}, s\right),
\end{aligned}
$$

for the iteration, which continues until $\max _{i=1, N+1}\left\{\left|\delta w_{i}\right|\right\}<10^{-5}$, where

$$
\begin{aligned}
& \mathscr{F}_{w}(w, s)=\left\{\begin{array}{cc}
G_{u}(u, \lambda) & G_{\lambda}(u, \lambda) \\
\dot{u}\left(s_{o}\right)^{T} & \dot{\lambda}\left(s_{o}\right)
\end{array}\right\}, \\
& F_{s}(w, s)=\left[\begin{array}{c}
0 \\
-1
\end{array}\right] .
\end{aligned}
$$

If the initial guess is close enough to the solution the iterates converge at a turning point because $\mathscr{F}_{w}(w, s)$ is not singular at that point, and moreover subcritical and supercritical bifurcation points can be easily overpassed. When subcritical or supercritical bifurcations are encountered the bifurcated branch can be computed merely by changing the tangent vector used in Eq. (11) as follows:

$$
N_{2}(w, s)=\psi^{T}\left[w(s)-w\left(s_{o}\right)\right]-\Delta s=0,
$$

$\psi$ being the critical unit eigenvector of $\mathscr{F}_{w}\left(s_{o}\right)$. The initial guess is now obtained by

$$
w^{0}=w_{o}+\psi \Delta s,
$$

and Eq. (20) substitutes Eq. (11) in the following iterates.

Equations (1)-(9) are linearized around a known solution by seeking solutions of the form

$$
\begin{aligned}
& F=F_{o}+f+o\left(\left|\frac{f}{F_{o}}\right|\right), \quad \text { where }\left|\frac{f}{F_{o}}\right|<1, \\
& \left.X=X_{o}+\chi+o\left(\mid \frac{\chi}{X_{o}}\right)\right), \quad \text { where }\left|\frac{\chi}{X_{o}}\right|<1, \\
& P=P_{o}+p+o\left(\left|\frac{p}{P_{o}}\right|\right), \quad \text { where }\left|\frac{p}{P_{o}}\right|<1 .
\end{aligned}
$$

The leading terms obtained for $\mathrm{Eq}$. (1) are

$$
\begin{gathered}
\tilde{O}^{-3 / 2}\left[\tilde{A}+\left(\tilde{B}-\frac{3 \tilde{A} \tilde{Q}}{2 \tilde{O}}\right) f+\left(\tilde{C}-\frac{3 \bar{A} \bar{S}}{2 \bar{O}}\right) f_{z}+\left(\bar{D}-\frac{3 \tilde{A} \tilde{T}}{2 \tilde{O}}\right) f_{\theta}\right. \\
\left.+\tilde{E} f_{z \bar{\delta}}+\tilde{G} f_{\theta \theta}+\tilde{H} f_{z \theta}+\left(\tilde{R}-\frac{3 \bar{A} \bar{K}}{2 \tilde{O}}\right) \chi_{z}+\tilde{L}_{X z z}\right]+P_{o} \\
+p-z B_{a}+B_{l}\left(F_{o}+f\right) \cos \theta+B_{l}\left(X_{o}+\chi\right)=0,
\end{gathered}
$$

where $\tilde{A}, \tilde{B}, \tilde{C}, \tilde{D}, \tilde{E}, \tilde{G}, \tilde{H}, \tilde{K}, \tilde{L}, \tilde{O}, \tilde{Q}, \tilde{R}, \tilde{S}$, and $\tilde{T}$ are known functions of $F_{o}, X_{o}$, and $P_{o}$ and consequently of the variables $z$ and $\theta$ (additional details on these functions can be obtained upon request to the authors). The leading terms obtained for the boundary conditions are

$$
\begin{aligned}
& \int_{-\Lambda}^{\Lambda} d z \int_{0}^{2 \pi} F_{o}(z, \theta)^{2} d \theta \\
& \quad+2 \int_{-\Lambda}^{\Lambda} d z \int_{0}^{2 \pi}\left[F_{o}(z, \theta) f(z, \theta)\right] d \theta=4 \pi \Lambda V,
\end{aligned}
$$

$F_{o}( \pm \Lambda)+f( \pm \Lambda)-1=0$,

$X_{o}( \pm \Lambda)+\chi( \pm \Lambda)=0$,

$f(z, \theta)=f(z, \theta+2 \pi)$,

$f(z, 0)=f(z, \pi)$,

where, if $\left(F_{o}, X_{o}, P_{o}\right)$ is an exact solution of the problem, Eqs. (25)-(30) can be simplified, but all the terms have been retained because $\left(F_{o}, X_{o}, P_{o}\right)$ will only be an approximation to the solution in the iterative scheme.

\section{NUMERICAL METHOD}

In order to develop a centered finite difference scheme the domain has been characterized by a mesh, defined as the intersection of the free surface with the following planes:

$$
\begin{aligned}
& z=\Lambda\left(\frac{2 j}{J}-1\right), \quad j=0,1, \ldots, J, \\
& \theta=\frac{2 \pi}{I+1} i, \quad i=0,1, \ldots, I .
\end{aligned}
$$

A number of fictitious points are to be adjoined to the ones already defined, and they correspond to the intersection of the "continuation" of the liquid bridge beyond the disks, with the planes corresponding to

$$
j=-1, J+1, \quad i=0,1, \ldots, I .
$$

The linearized finite equations can be written as follows:

$$
\begin{aligned}
& \alpha_{i j} f_{j}^{i}+\beta_{i j} f_{j-1}^{i}+\gamma_{i j} f_{j+1}^{i}+\delta_{i j} f_{j}^{i-1}+\varphi_{i j} f_{j}^{i+1} \\
& \quad+\phi_{i j}\left(f_{j+1}^{i+1}-f_{j+1}^{i-1}-f_{i-1}^{i+1}+f_{j-1}^{i-1}\right)+\mu_{i j} \chi_{j-1}+\nu_{i j} \chi_{j} \\
& \quad+\rho_{i j} \chi_{j+1}+p=\psi_{i j}, \quad i=0, \ldots, I, \quad j=0, \ldots, J, \\
& f_{J}^{i}=1-F_{o J}^{i}, \quad i=1, \ldots, I, \\
& f_{0}^{i}=1-F_{o 0}^{i}, \quad i=1, \ldots, I, \\
& \chi_{J}=-X_{o J}, \\
& \chi_{0}=-X_{00}, \\
& f_{j}^{0}-f_{j}^{l+1}=0, \quad j=-1, \ldots, J+1, \\
& f_{j}^{0}-f_{j}^{(I+1) / 2}=0, \quad j=-1, \ldots, J+1,
\end{aligned}
$$

where the coefficients $\alpha_{i j}, \beta_{i j}, \gamma_{i j}, \delta_{i j}, \varphi_{i j}, \phi_{i j}, \mu_{i j}, \nu_{i j}$, $\rho_{i j}$, and $\psi_{i j}$ are functions of the values $F_{o j}^{i}, X_{o j}$, and $P_{a}$.

The equation of conservation of the volume has been discretized using the alternative extended Simpson's rule $e^{14}$ when integrating in the axial direction, and the extended trapezoidal rule ${ }^{14}$ when integrating in the azimuthal direction, to obtain 


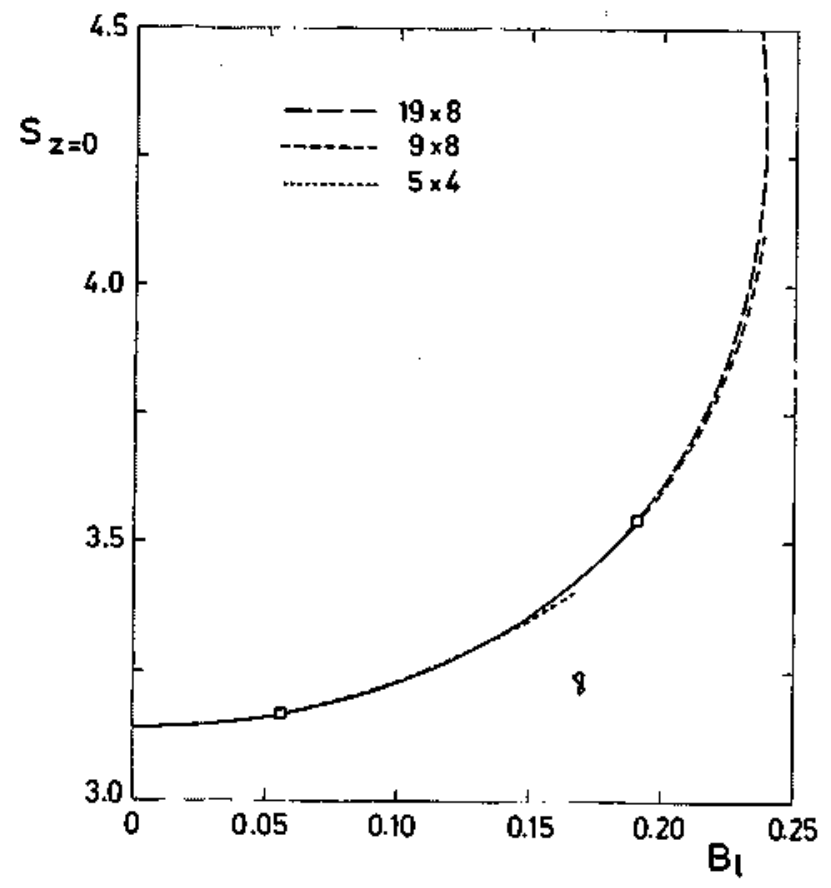

FIG. 2. Main branch of the bifurcation diagram for a liquid bridge of cylindrical volume and $\Lambda=2.8$. The area of section $z=0$ is presented versus the lateral Bond number. Stable branches are plotted with solid lines and unstable branches are plotted with dashed lines. The mesh size is expressed as $I \times J$ in the legend.

$$
\sum_{i=0}^{I} \sum_{j=0}^{J} a_{i j} f_{j}^{i}=A .
$$

The set of linear algebraic equations has been solved by the Crout's method with partial pivoting ${ }^{14}$ and the critical eigenvector, $\psi$, has been obtained by the inverse iteration method. ${ }^{14}$

The size of the mesh has been chosen, analyzing the results obtained by meshes of different sizes when the varying natural parameter was the lateral Bond number. The critical values of $B_{1}$ for liquid bridges of different slendernesses were sought, and it was found that a minimum number of points in the mesh is needed to be able to find the complete bifurcation diagram, and this minimum increases as the slenderness of the liquid bridge decreases.

The bifurcation diagram obtained consists of a main branch of solutions with a fold point and a bifurcated branch of solutions, which, depending on the slenderness of the liquid bridge, bifurcates before or after reaching the fold point.

Figure 2 shows the main branch of the bifurcation diagram for a liquid bridge with $\Lambda=2.8$ and cylindrical volume, $V=1$, computed using meshes of different sizes. Stable branches are represented with a solid line. It can be appreciated that the algorithm is not capable of finding the turning point by using coarse meshes.

When progressing along the main branch a turning point is encountered, and after overpassing it the lateral Bond number decreases until it gets to be $B_{l}=0$ and its corresponding equilibrium shape is axisymmetric once again (this

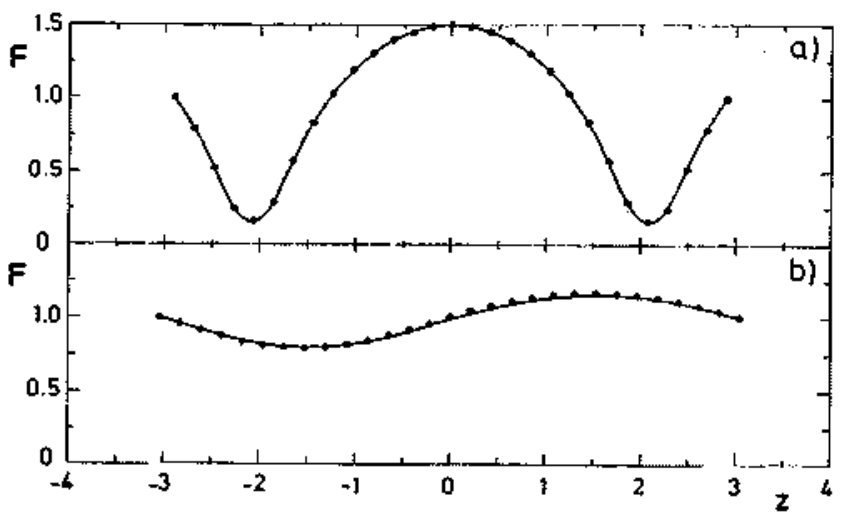

FIG. 3. Axisymmetric equilibrium shapes of cylindrical volume liquid bridges, with (a) $\Lambda=2.9$ progressing along the main brancb of solutions, (b) $\Lambda=3.05$ progressing along the bifurcated branch of solutions. Results $0 b$ tained by (O) a mesh $I \times J=19 \times 28$; ( $\rightarrow$ ) analytical solution.

shape has also been calculated in terms of elliptic integrals using the formulation described by Martínez and Perales ${ }^{15}$ ). The equilibrium shapes calculated by both methods are represented in Fig. 3(a) for a cylindrical volume liquid bridge with $\Lambda=2.9$. In the same way when progressing along the bifurcated branch the lateral Bond number decreases until for $B_{l}=0$ an axisymmetric equilibrium shape is encountered; again this shape can be calculated in terms of elliptic integrals. The axisymmetric equilibrium shapes of a cylindrical volume liquid bridge with $\Lambda=3.05$ calculated by both methods are represented in Fig. 3(b).

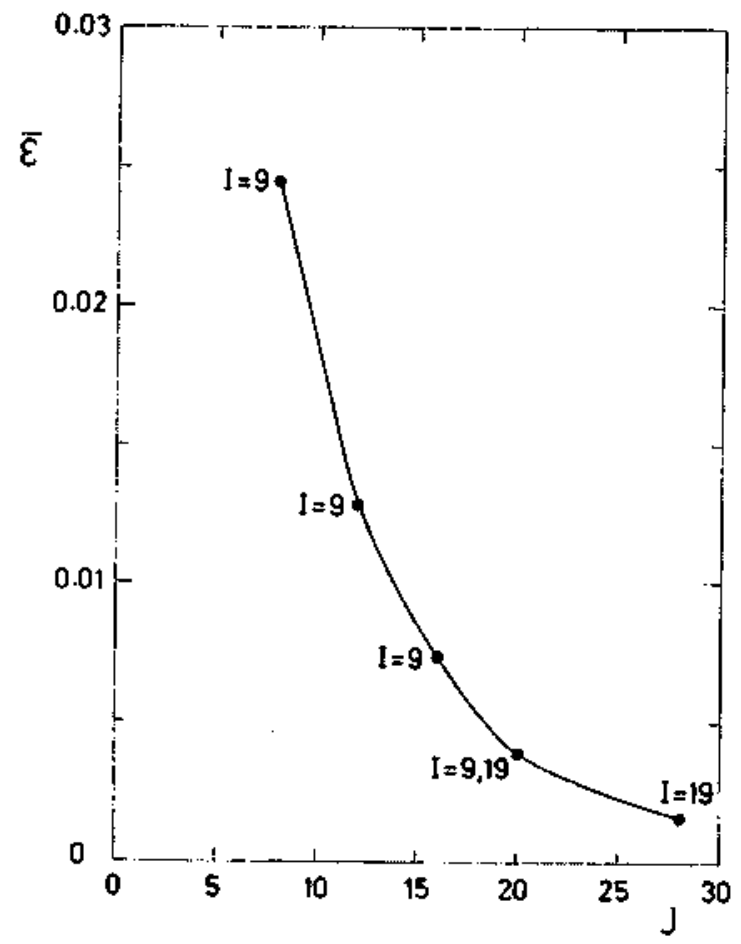

FIG. 4. Error made calculating axisymmetric equilibrium shapes as a function of the number of points in the mesh of a cylindrical volume liquid bridge of $\Lambda=2.9$. 
The axisymmetric shapes obtained progressing along the main branch for a cylindrical volume liquid bridge with $\Lambda=2.9$ has been calculated by meshes of different sizes and compared with the one obtained in terms of elliptic integrals by using as a measure of the error:

$$
\bar{\epsilon}=\frac{1}{(J+1)} \sqrt{\sum_{j=0}^{J}\left(F_{j}^{0}-F_{j}^{*}\right)^{2}},
$$

where $F^{*}$ is the expression obtained in terms of elliptic integrals. This error parameter is represented in Fig. 4 as a function of the number of points in the mesh.

It can be deduced that the number of points needed to overpass the turning point increases as the slenderness decreases. The number of points in the axial direction of the mesh has a greater influence in the results than the number of points in the azimuthal direction. The size of the mesh chosen to obtain the results presented in this paper was $I \times J$ $=19 \times 28$. Anyway, in order to obtain results for liquid bridges with small slenderness $(V=1.0, \Lambda \leqslant 2.0)$, a bigger mesh was used, $I \times J=19 \times 36$.

The dimensionless potential energy of the equilibrium shapes has the following form:

$$
\mathscr{H}=\int_{\Omega}\left[B_{a} z-B_{l} x\right] d \Omega+\Gamma,
$$

where the dimensionless potential energy has been defined as $G B=G / /\left(\sigma R_{0}^{2}\right)$ (where $\bar{Z} b$ is the potential energy of the configuration), $\Gamma$ is the dimensionless area of the free surface, and $\Omega$ is the domain occupied by the liquid bridge.

The above equation can be expressed in a simpler way as

$$
\begin{aligned}
& \theta b=\frac{B_{l}}{2} \int_{0}^{2 \pi} \int_{-\Lambda}^{\Lambda} X(z) F^{2}(z, \theta) d z d \theta \\
& +\frac{B_{l}}{3} \int_{0}^{2 \pi} \int_{-\mathrm{A}}^{\mathrm{A}} F^{3}(z, \theta) \cos \theta d z d \theta \\
& -\frac{B_{a}}{2} \int_{0}^{2 \pi} \int_{-\Lambda}^{\Lambda} F^{2}(z, \theta) z d z d \theta+\Gamma .
\end{aligned}
$$

The bidimensional integrals present in Eq. (44) have been calculated using the alternative extended Simpson's rule $^{14}$ when integrating in the axial direction and the extended trapezoidal rule ${ }^{14}$ when integrating in the azimuthal direction.

\section{RESULTS}

In the following the influence of a lateral gravity and a lateral and an axial gravity combined is analyzed for liquid bridges, with $\Lambda \geq 1.8$. The lateral gravity is a symmetric perturbation about the $z=0$ plane, therefore its effect is analyzed by itself and also combined with an axial gravity in order to break this symmetry.

\section{A. Absence of axial gravity}

It is known that there is a critical slenderness for long cylindrical liquid bridges for which a subcritical bifurcation is encountered, so that for $\Lambda$ values smaller than critical, the

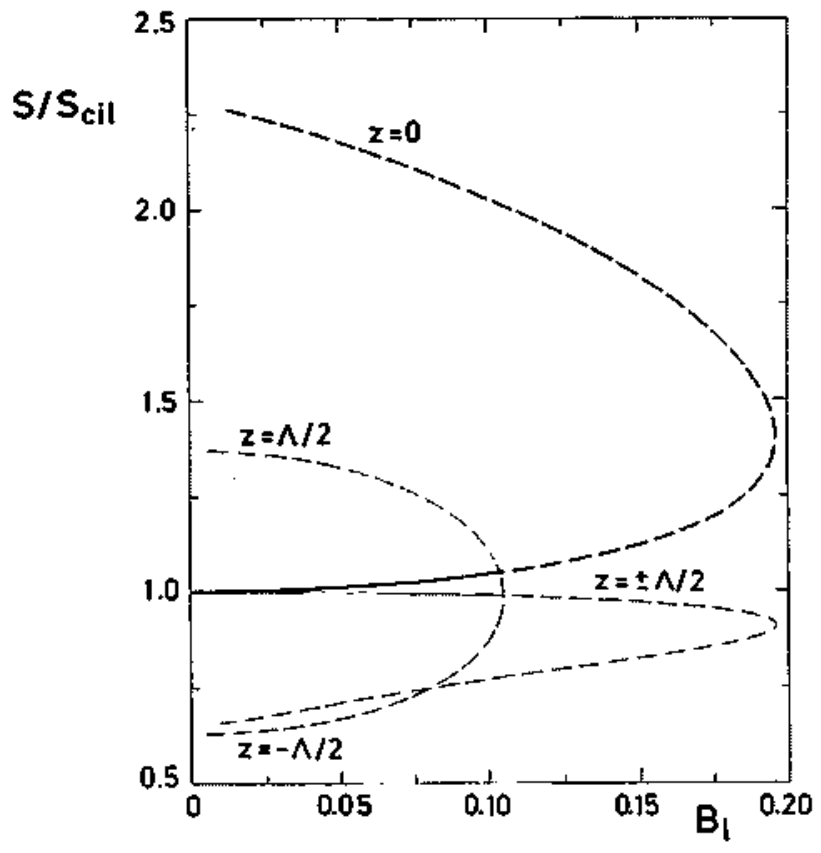

FIG. 5. Bifurcation diagram of a cylindrical volume liquid bridge with $\Lambda=3.05$. The areas of sections $z=+\Lambda / 2$ and $z=0$ are represented versus the lateral Bond number.

Liquid bridges are stable. ${ }^{5}$ The results obtained here confirm the existence of this bifurcation for long liquid bridges, but it is observed that for short ones the character of the destabilization varies because it is due to a turning point instead of to a subcritical bifurcation point. Cylindrical volume liquid bridges subject to a lateral gravity with $\Lambda>2.38$ will destabilize as a consequence of a subcritical bifurcation point which associated eigenfunction is antisymmetric about the $z=0$ plane, so that the breaking process will probably lead to a nonsymmetric configuration of two drops of different volume. On the other hand, if $\Lambda<2.38$ the destabilization is determined by a turning point and its associated eigenfunction is symmetric about the $z=0$ plane so that the breaking process will probably lead to a symmetric configuration of two equal drops.

The character of the breaking process described is quite similar to the one obtained by decreasing the volume of the bridge, ${ }^{16.17}$ and for cylindrical isorotating liquid bridges in the absence of gravity. 8

The bifurcation diagram obtained by representing the areas of the sections $z= \pm \Lambda / 2$ and $z=0$ as a function of the lateral Bond number for a cylindrical volume liquid bridge with $\Lambda=3.05$ is shown in Fig. 5. The unstable branches are represented with dashed lines and the stable ones with solid lines.

The bifurcation diagrams for different slenderness are very similar to the preceding one. As the slenderness decreases, being $\Lambda>2.38$, the turning point is encountered for increasing values $B_{l}$ and the subcritical bifurcation point draws near the turning point until for $\Lambda=2.38$ both coincide. If $\Lambda<2.38$ the subcritical bifurcation is encountered, progressing along the main branch of solutions, after the turning 


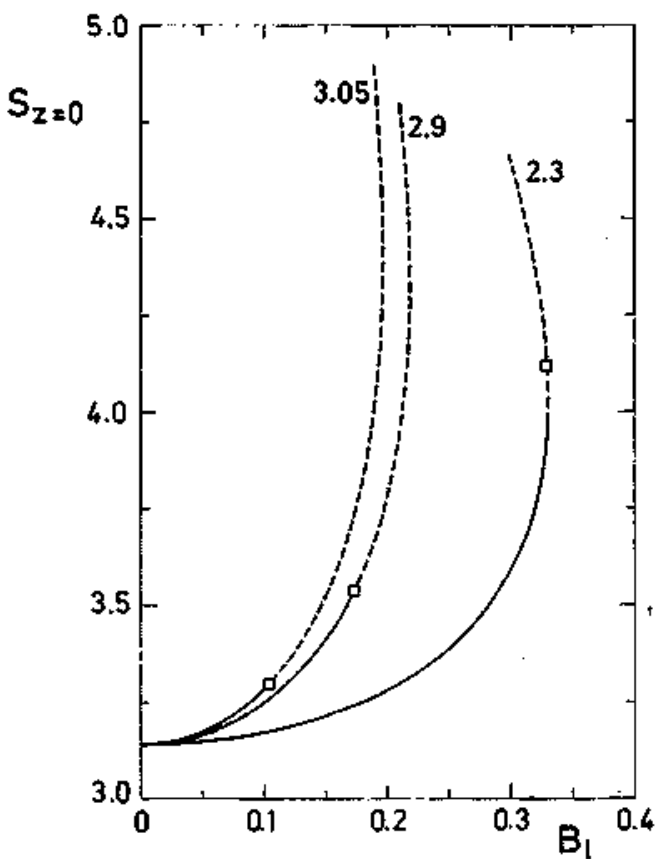

FIG. 6. Main branches of the bifurcation diagrams for cylindrical volume liquid bridges with $\Lambda=2.3,2.9$, and 3.05 . The area of section $z=0$ is represented versus the lateral Bond number. Stable branches are plotted with solid lines and unstable branches are plotted with dashed lines. The subcritical bifurcation point is pointed out with a square.

point. As $\Lambda$ decreases the turning point occurs for increasing values of $B_{l}$, the same as the subcritical bifurcation point, but these two points move away from each other.

Figure 6 shows the influence of the slenderness in the main branch of the bifurcation diagram. The area of section $z=0$ is represented versus the lateral Bond number, the stable branches are represented with solid lines and the unstable with dashed lines, and the position of the subcritical bifurcation points have been pointed out with a square.

In the plane of the parameters $\left(\Lambda, B_{l}\right)$ the profiles describing the turning and the subcritical bifurcation points for cylindrical volume liquid bridges are tangent when $\Lambda=2.38$, where the character of the instability changes. These profiles are shown in Fig. 7. The portion of the curves that determine the stability limit is represented by solid lines.

Comparing in Fig. 8 the critical slenderness obtained here, with the one obtained by Coriel et al. ${ }^{4}$ and Perales using asymptotic techniques,

$$
\Lambda \approx \pi\left(1-\frac{\pi^{2}}{4} B_{l}^{2}\right),
$$

it can be seen how these results agree extremely well for the nonsymmetric destabilization and how the disagreement increases as the slenderness decreases, as expected, because the asymptotic results were developed around a slenderness $\Lambda=\pi$.

The quasistatic evolution of the equilibrium shape of a cylindrical volume liquid bridge with $\Lambda=3.05$ along the main branch of solutions is represented in Fig. 9, while the quasistatic evolution of the equilibrium shapes of the same

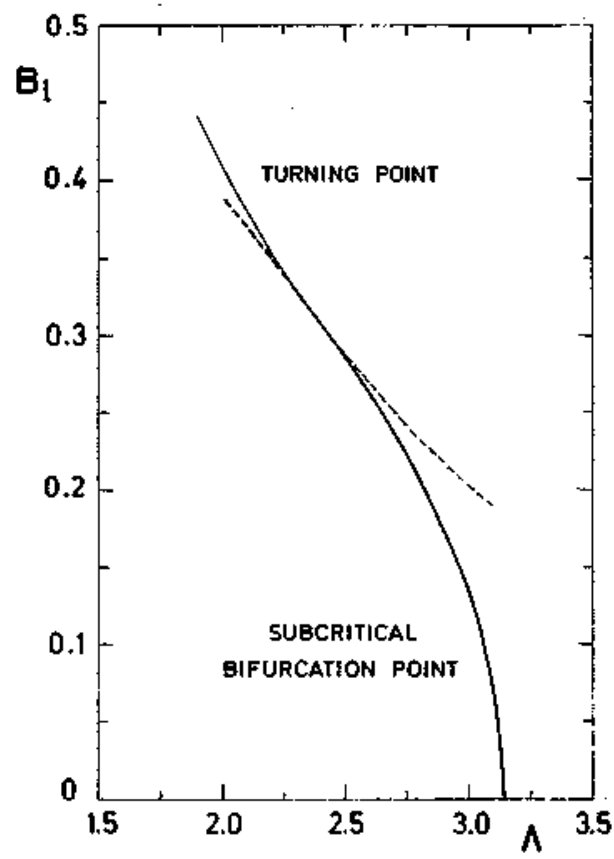

FIG. 7. Subcritical bifurcation point and turning point of a cylindrical volume fiquid bridge versus the slenderness. The portion of the profiles determining the stability limit are represented by soljd lines.

liquid bridge along the bifurcated branch is represented in Fig. 10. The shapes obtained when progressing along the main branch are symmetric about the $z=0$ plane and present two necks that become narrower as $B_{l}$ evolves. The shapes

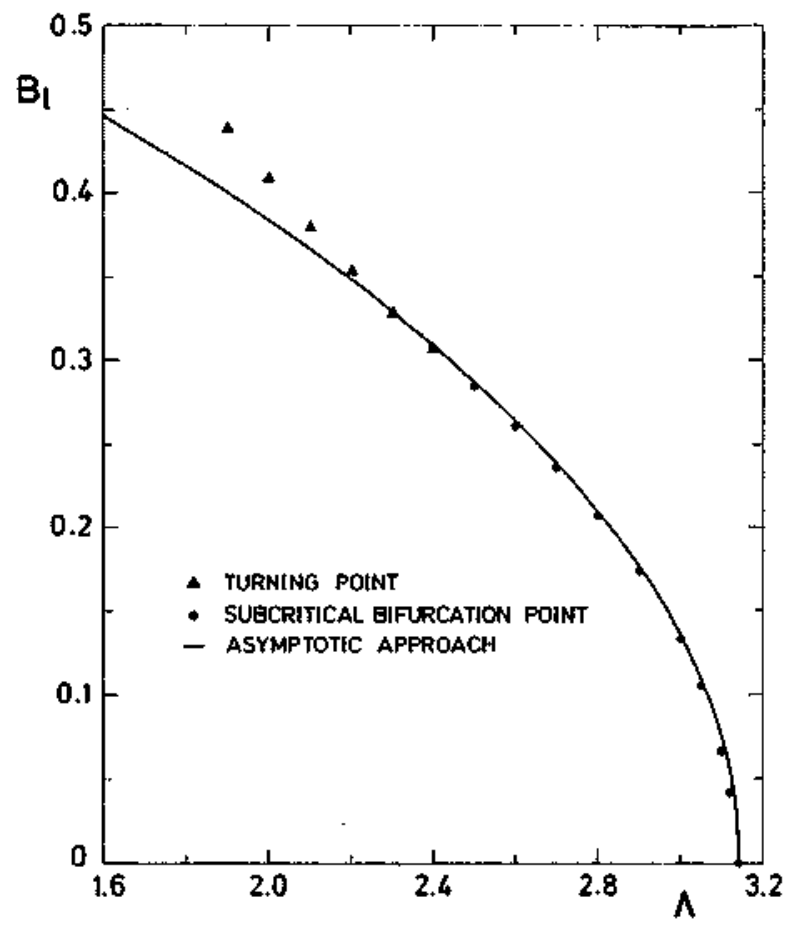

FIG. 8. Stability limits of a cylindrical liquid bridge. (-) Asymptotic approach; ${ }^{4,5}(\Delta)$ turning point, $(\boldsymbol{O})$ subcritical bifurcation point. 


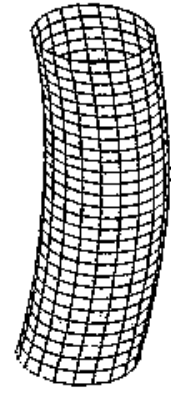

a)

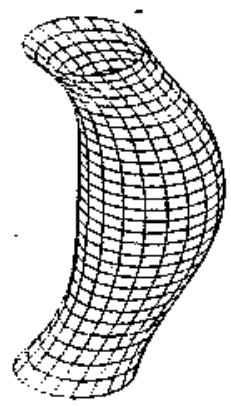

d)

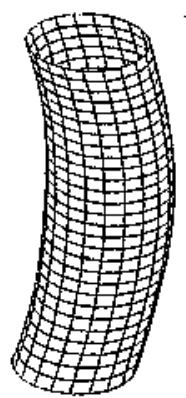

b)

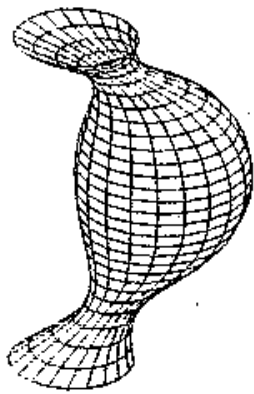

e)

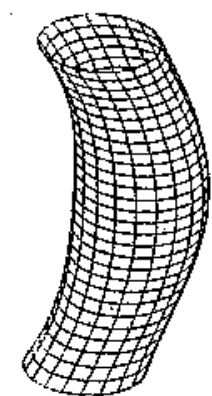

c)

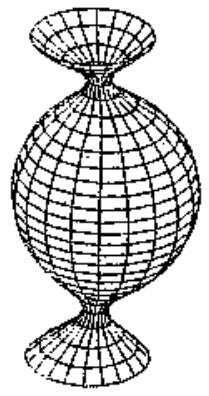

f)
FIG. 9. Quasistatic evolution of the equilibrium shapes of a cylindrical volume liquid bridge with $\Lambda=3.05$ along the main branch of solutions. The lateral Bond number corresponding to eact stape is (a) 0.0827 , (b) 0.1084 , (c) 0.1646 , (d) 0.1956 , (e) 0.1126 , and (f) 0.0101 . Shape (a) is stable and the rest are unstable.

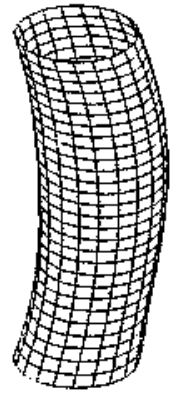

a)

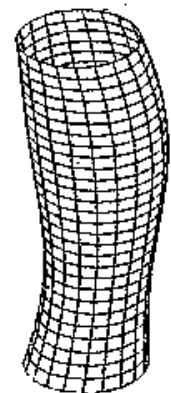

c)

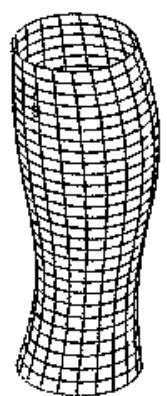

d)

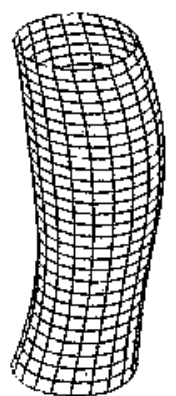

b)

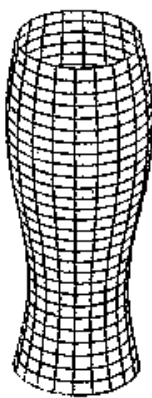

e)
FIG. 10. Quasistatic evolution of a cylindtical volume liquid bridge with $\Lambda=3.05$ ajong the bifurcated branch of solutions. The lateral Bond number corresponding to each shape is (a) 0.1014 , (b) 0.0881 , (c) 0.0732 , (d) 0.0515 , and (e) 0.0049 .

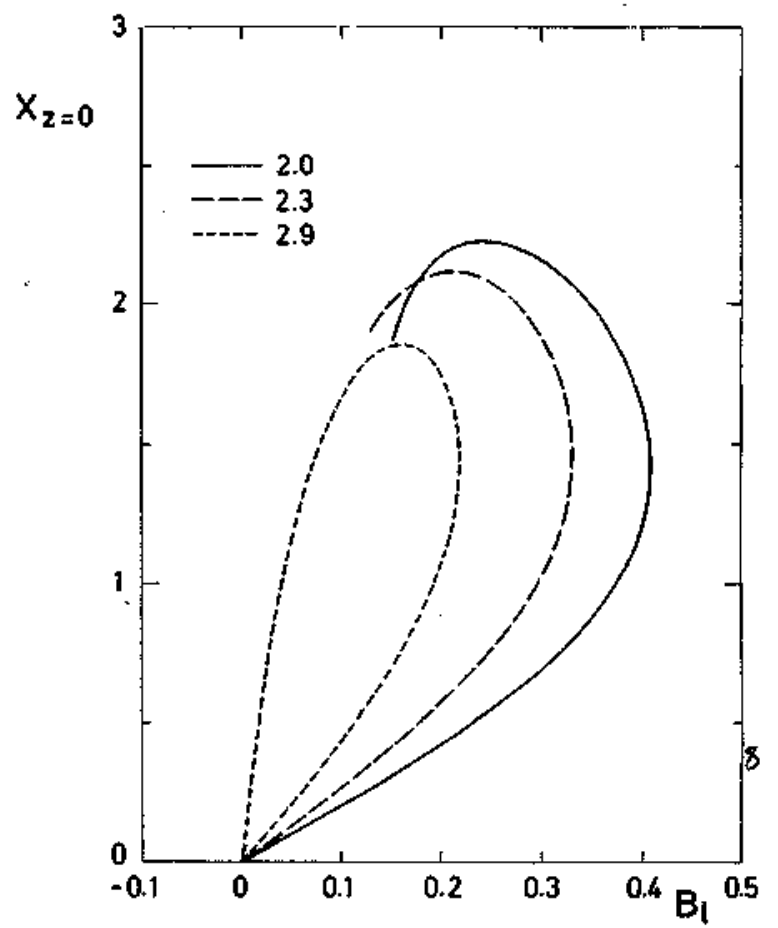

FIG. 11. Evolution of $X_{z=0}$ atong the majn branch of solutions for three cylindrical volume liquid bridges with $\Lambda=2.0,2.3$, and 2.9 .

obtained progressing along the bifurcated branch present one neck that also becomes narrower as $B_{l}$ decreases.

The maximum value of $|X(z)|,\left|X_{z=0}\right|$, increases while progressing along the main branch until a maximum is reached (this maximum increases as the slenderness decreases), from this point on $\left|X_{\mathrm{z}=n}\right|$ decreases until it gets to be $X \equiv 0$ for $B_{l}=0$, and an axisymmetric unstable solution is encountered. Figure 11. shows $X_{z=0}$ as a function of the lateral Bond number for cylindrical volume liquid bridges with $\Lambda=2.0,2.3$, and 2.9 .

The bifurcation diagrams obtained for liquid bridges of arbitrary volume are very similar to the ones obtained for cylindrical volume liquid bridges. As the volume increases, the turning point occurs for decreasing values of the lateral Bond number. The variation of the position of the subcritical bifurcation point with the volume is not so easy to describe, but the main branch of the bifurcation diagram for three liquid bridges with $\Lambda=2.9$ and $V=1.0,1.1$, and 1.2 , in which the area of section $z=\Lambda / 2$ has been plotted as a function of the lateral Bond number, can be seen in Fig. 12. The stable branches are represented with solid lines, while the instable ones are represented with dashed lines. The curves determining the stability limits in the plane $\left(\Lambda, B_{l}\right)$ for liquid bridges with $V=0.6,0.7,0.8,0.9,1.0,1.1$, and 1.2 are represented in Fig. 13. These stability limits are also represented in the plane $(\Lambda, V)$ for constant values of the lateral Bond number, in Fig. 14.

\section{B. Influence of an axial gravity}

As expected, when introducing a small axial gravity the character of the solution changes completely, due to the loss 


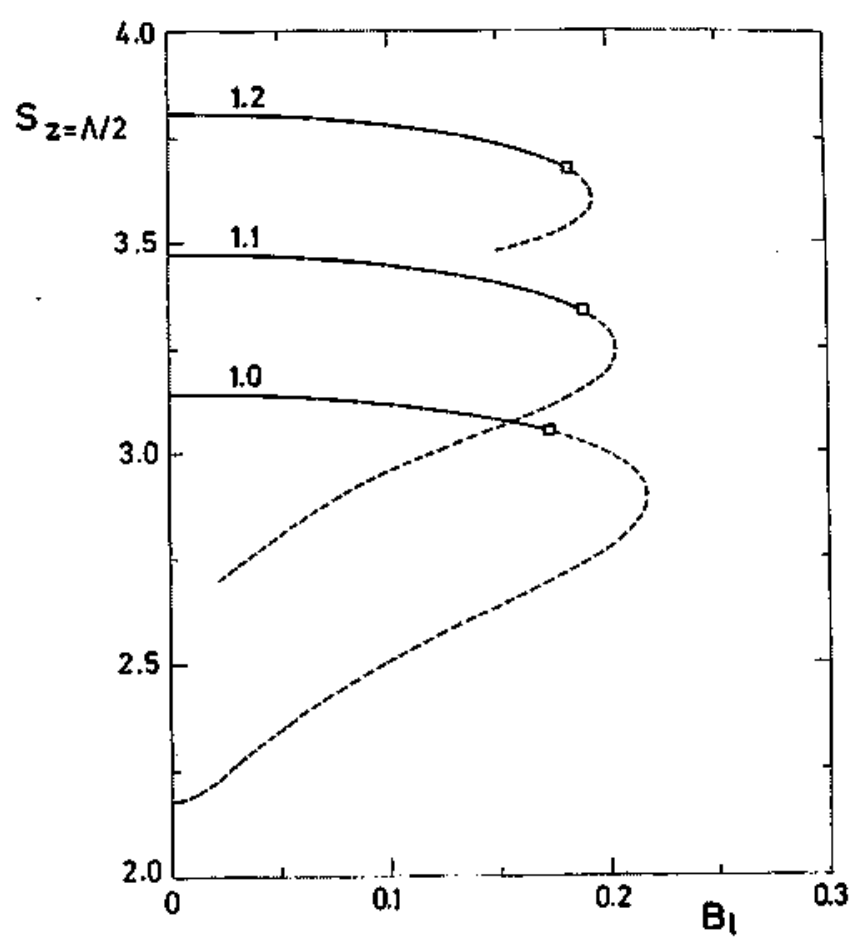

FIG. 12. Main branch of the bifurcation diagram for three liquid bridges with $\Lambda=2.9$ and $V=1.0,1.1$, and 1.2. The area of section $z=\Lambda / 2$ is plotted against the lateral Bond number. Stable branches are plotted with solid lines and unstable branches are plotted with dashed lines. The subcritical bifurcation point is pointed out with a square.

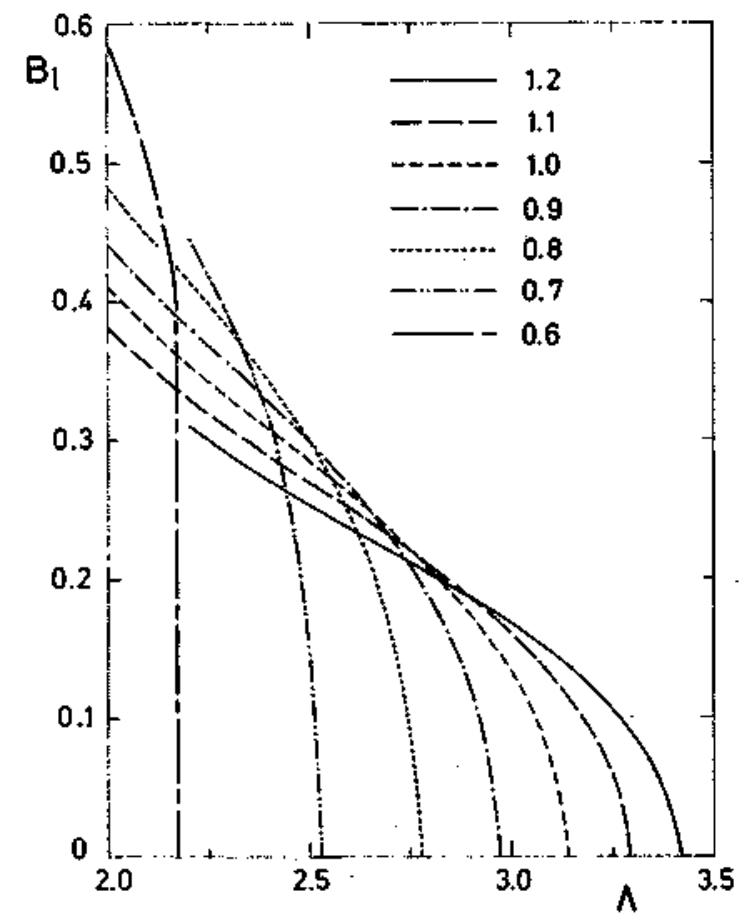

FIG. 13. Stability limits for liquid bridges with different volumes. Maximum value of the lateral Bond number that a liquid bridge of constant volume (indicated in the legeed) can withstand as a function of its slenderness.

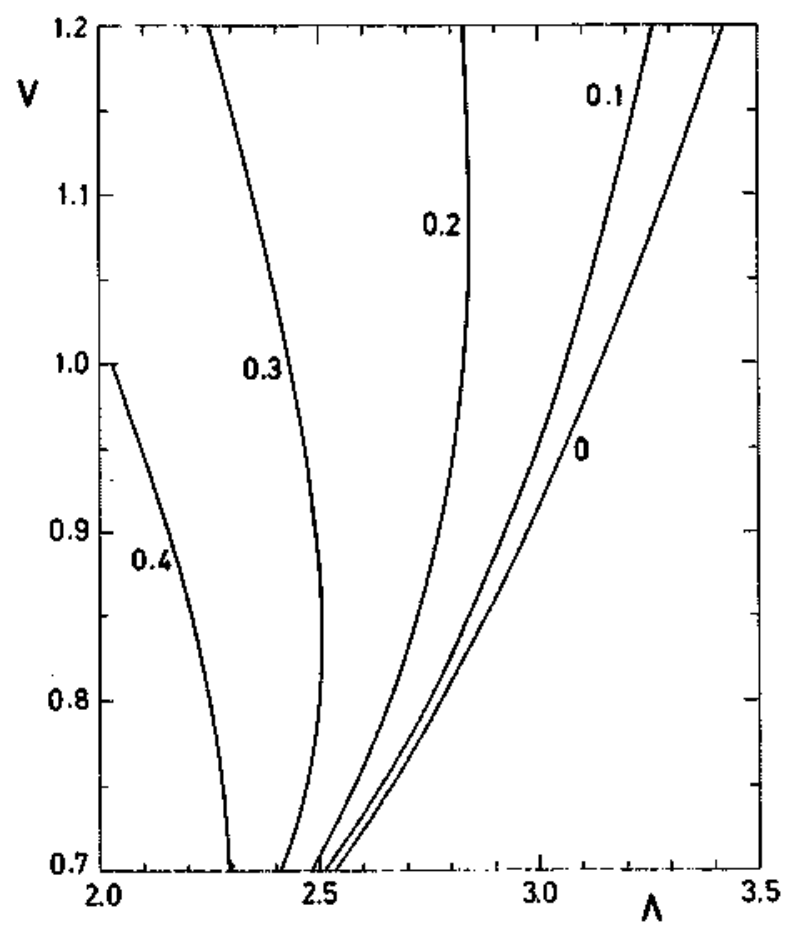

FIG. 14. Stability limits of liquid bridges subjected to different values of the Jateral Bond number (indicated on the curves).

of symmetry of the configuration about the plane $z=0$. The critical point determining the instability of the solutions when progressing in the lateral Bond number is always a turning point. There does not exist a subcritical bifurcation point for long bridges, as occurred when the axial Bond number was zero. As $B_{a}$ increases the critical $B_{l}$ decreases. Figure 15 represents the area of sections $z= \pm \Lambda / 2$ and $z=0$ as a function of the lateral Bond number for a cylindrical volume liquid bridge, with $\Lambda=2.9$ and $B_{a}=0.003$. The quasistatic evolution of this liquid bridge is represented in Fig. 16.

The stability limits of cylindrical volume liquid bridges subjected to different values of $B_{a}$ are represented in the plane $\left(\Lambda, B_{l}\right)$ in Fig. 17. It can be observed from this figure that when applying a small $B_{a}$ the critical $B_{l}$ does not change too much for small values of the slenderness. In fact, the curve corresponding to $B_{a}=10^{-3}$ is indistinguishable from the one corresponding to $B_{a}=0$ for values of the slenderness $\Lambda<2.3$. Nevertheless, as the slenderness of the liquid bridge increases, the critical values of $B_{l}$ when $B_{a} \neq 0$ differ more from the ones corresponding to $B_{a}=0$.

The described behavior was already obtained approximately for liquid bridges of slendernesses around $\Lambda=\pi$ using asymptotic techniques. ${ }^{4,5}$ There the critical slenderness deduced

$$
\Lambda=\pi\left[1-\left(\frac{3}{2}\right)^{4 / 3} B_{a}^{2 / 3}-\frac{\pi^{2}}{4} B_{l}^{2}\right],
$$

is represented in Fig. 18 for $B_{a}=10^{-2}$ and $B_{a}=10^{-3}$ along with the results obtained here. 


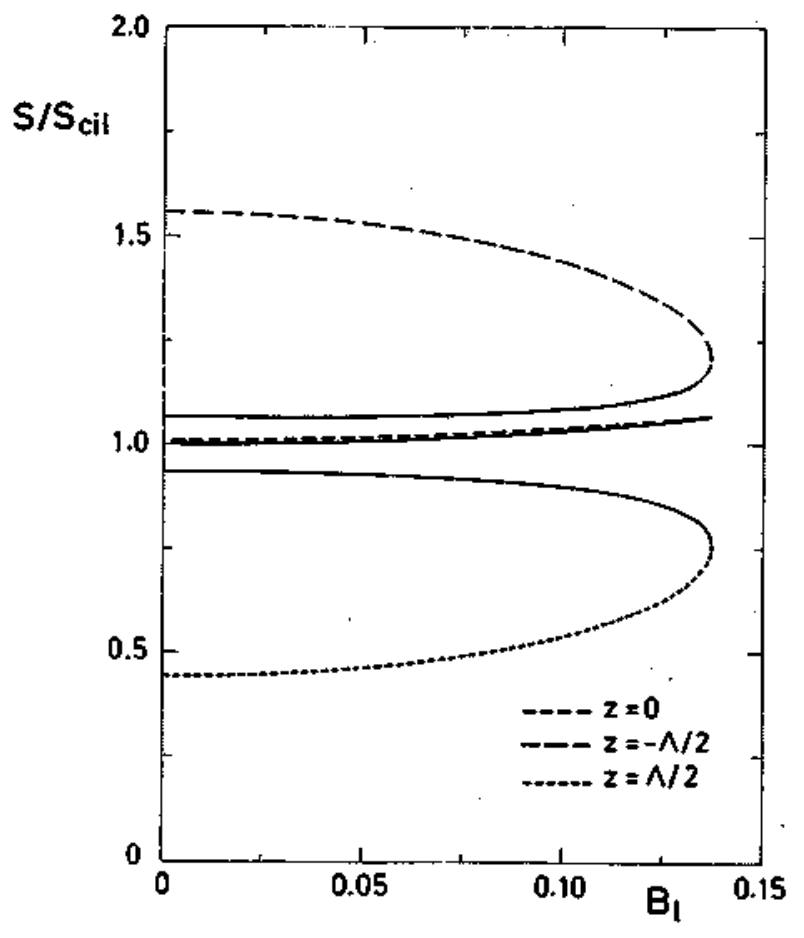

FIG. 15. Area of sections $z= \pm \Lambda / 2$ and $z=0$ as a function of the lateral Bond number applied to a cylindrical volume liquid bridge with $A=2.9$ and $B_{a}=0,003$. Stable branches are pjotted with solid lines and unstable branches are plotted with dashed lines.

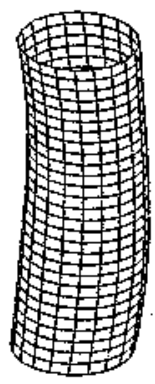

a)

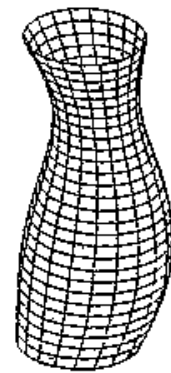

d)

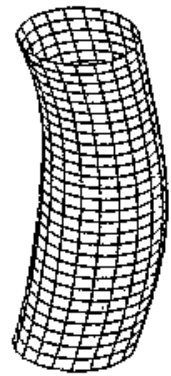

b)

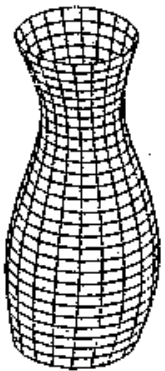

e)

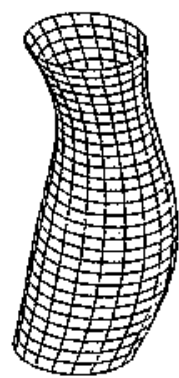

c)

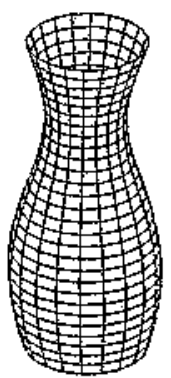

f)
FIG. 16. Quasistatic evolution of the equilibrium shapes of a cylindrical volume liquid bridge with $\Lambda=2.9$ and $B_{\mathrm{a}}=0.003$ when progressing in $B_{I}$. The corresponding lateral Bond numbers are (a) 0.0501 , (b) 0.1165 , (c) 0.1211 , (d) 0.0809 , (e) 0.0375 , and (f) 0.0075 .

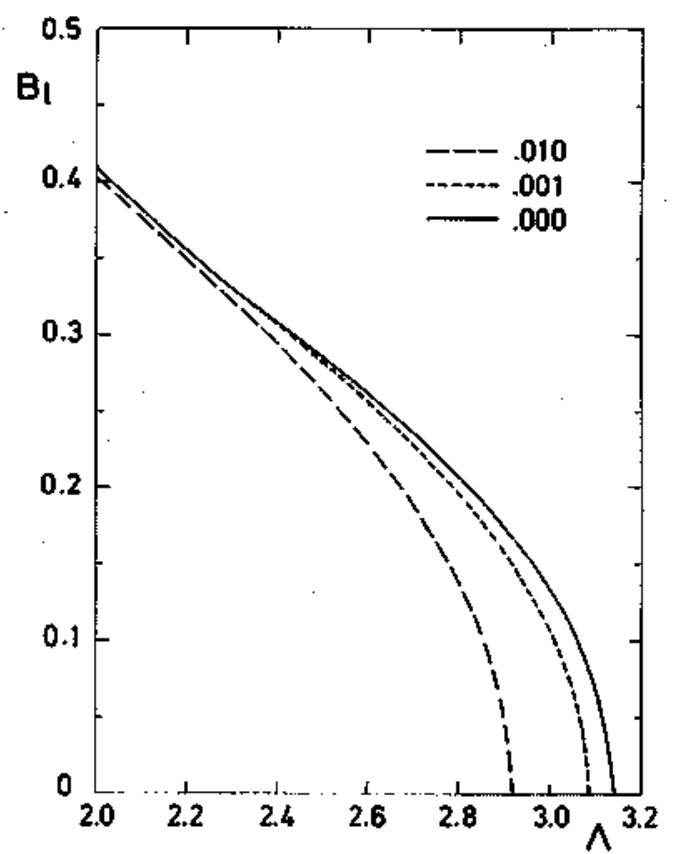

FIG. 17. Maximum value of the lateral Bond number that a cylindrical volume liqujd bridge, subjected to a constant value of $B_{a}$ (indicated in the legend), can withstand as a function of the slenderness.

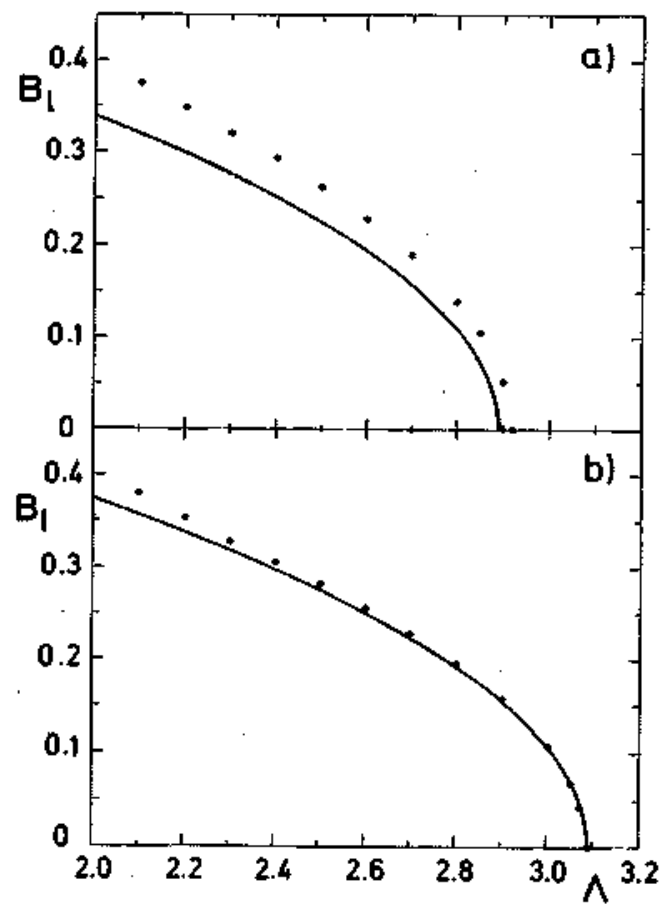

FIG. 18. Maximum value of the lateral Bond number that a cylindrical volume liquid bridge subjected to (a) $B_{a}=10^{-2}$ and (b) $B_{a}=10^{-3}$ can withstand as a function of the slenderness. (--) Asymptotic results; (O) numeric results. 


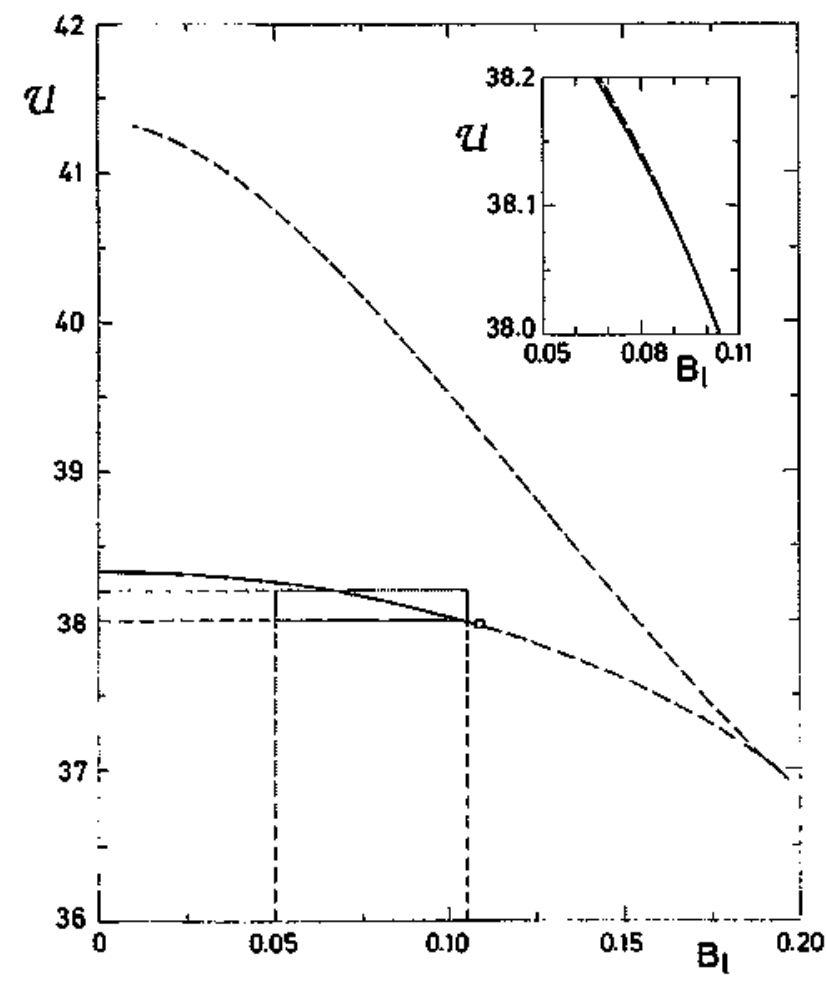

FIG. 19. Potential energy of the equilibrium shapes corresponding to the main and bifurcated branches of solutions of the bifurcation diagram of a cylindrical volume liquid bridge with $A=3.05$ as a function of the lateral Bond number. Stable branches are plotted with solid lines and unstable branches are plotted with dashed lines. A detait of the main and bifurcated branch is also plotted.

\section{Energy of the equilibrium shapes}

The potential energy of each one of the equilibrium shapes of a family of solutions obtained varying the lateral Bond number has been calculated, and, as expected, a cusp point has been obtained at the critical points.

The potential energy of the equilibrium shapes corresponding to the main and bifurcated branches of solutions obtained for a cylindrical volume liquid bridge with $\Lambda=3.05$ as a function of the lateral Bond number is represented in Fig. 19. The stable branches of solutions are represented with solid lines, while the unstable ones are represented with dashed lines.

\section{CONCLUSIONS}

The developed algorithm has proved to be a useful tool in calculating families of equilibrium shapes obtained by varying a parameter of the configuration. The influence of the parameter studied happened to be very close to that predicted by asymptotic methods, even in quite large ranges of the values of the parameters.

When studying the influence of lateral gravity, two kinds of instabilities were found, depending on the slenderness of the bridge. For long liquid bridges, the instability is due to a subcritical bifurcation point, and the resulting configuration after the breaking process is expected to be asymmetric about the plane $z=0$. For short liquid bridges the instability is due to a turning point and the resulting configuration expected after the breaking process is symmetric about $z=0$.

The introduction of a small axial gravity changes the structure of the solutions, so that they become asymmetric about $z=0$, and the instability is always due to a turning point. The influence of the axial gravity is more important for long bridges than for short ones. The configuration obtained after the breakage is expected to be asymmetric about $z=0$.

The developed algorithm can also be applied to other configurations, such as those with noncoaxial, noncircular, or nonparallel supporting disks.

\section{ACKNOWLEDGMENTS}

The authors are indebted to Professor J. M. Vega and Dr. F. J. Mancebo for their helpful discussions.

This work has been sponsored by the Spanish Comisión Interministerial de Ciencia y Tecnologia (CICXT) and is part of a more general endeavor for the study of fluid physics and materials in processing under microgravity (Project No. ESP92-0001-CP).

'J. A. F. Plateau, "Experimental and theoretical researches on the figures of equitibrium of a liquid mass withdrawu from the action of gravity," translated in the Annual Report of the Smithsonian Institute, 1863-1866, p. 207.

${ }^{2}$ A. D. Myshkis, V. G. Babskii, N. D. Kopachevskii, L. A. Slobozhanin, and A. D. Tyuptsov, Low-Gravity Fluid Mechanics (Springer-Verlag, Berlin, 1987).

${ }^{3}$ L. A. Slobozhanin and J. M. Perales, "Stability of liquid bridges between equal disks in an axial gravity field," Phys. Fluids A 5, 1305 (1993).

"S. R. Coriell, S. C. Hardy, and M. R. Cordes, "Stability of liquid zones," J. Colloid Interface Sci. 60, 126 (1977).

${ }^{5}$ J. M. Perales, "Non-axisymmetric effects on long liquid bridges," Acta Astronaut. 15, 561 (1987).

6J. Meseguer, N. A. Bezdenejoykh, J. M. Perales, and P. Rodríguez de Francisco, "Theoretical and experimental analysis of stability limits of non-axisymmetric liquid bridges," Microgravity Sci. Technol (in press).

${ }^{7}$ J. M. Perales, A. Sanz, and D. Rivas, "Eccentric rotation of a liquid bridge," Appl. Microgravity Technol. 2, 193 (1990).

${ }^{8} \mathrm{~J}$. M. Vega and J. M. Perales, "Almost cylindrical isorotating liquid bridges for small Bond numbers," in: ESA \$P-191, 1983, p. 247.

${ }^{9} R$. A. Brown and L. E. Scriven, "The shapes and stability of captive rotating drops," Philos. Trans. R. Soc. London Ser. A 297, 51 (1980).

${ }^{10}$ A. Lawal and R. A. Brown, "The stability of an inclined pendant drop," J. Colloid Interface Sci. 89, 332 (1982).

"A. Lawal and R. A. Brown, "The stability of an inclined sessile drop," J. Colloid Interface Sci. 89, 346 (1982).

${ }^{12} \mathrm{H}$. Chen and M. Z. Saghir, "Non-axisymmetric equilibrium shapes of the liquid bridge," Microgravity Sci. Technol. 7, 12 (1994).

${ }^{13}$ H. B. Keller, Lectures on Numerical Methods in Bifurcation Problems (Sptinger-Verlag, New York, 1987).

${ }^{14}$ W. H. Press, B. P. Flannery, S. A. Teukolsky, and W. T. Vettetling, Numerical Recipes in $C$ (Cambridge University Press, Cambridge, 1988).

${ }^{15}$ I. Martinez and J. M. Perales, "Liquid bridge stability data," J. Cryst. Growth 78, 369 (1986).

${ }^{16} \mathrm{~J}$. Meseguer, "The breaking of axisymmetric slender liquid bridges," $J$. Fluid. Mech. 130, 123 (1983).

${ }^{17} \mathrm{~A}$. Sanz and I. Martinez, "Minimum volume for a liquid bridge between equal disks," J. Colloid Interface Sci. 93, 235 (1983). 